\title{
Stokes Flow Past Three Spheres
}

\author{
Helen J. Wilson \\ Mathematics Department, University College London, Gower Street, London WC1E \\ $6 B T, U K$
}

\begin{abstract}
In this paper we present a numerical method to calculate the dynamics of three spheres in a quiescent viscous fluid. The method is based on Lamb's solution to Stokes flow and the method of reflections, and is arbitrarily accurate given sufficient computer memory and time. It is more accurate than multipole methods, but much less efficient. Although it is too numerically intensive to be suitable for more than three spheres, it can easily handle spheres of different sizes. We find no convergence difficulties provided we study mobility problems, rather than resistance problems.

After validating against the existing literature, we make a direct comparison with Stokesian Dynamics (SD), and find that the largest errors in SD occur at a sphere separation around 0.1 radius. Finally, we present results for an example system having different-sized spheres.
\end{abstract}

Keywords: Viscous flow, Spherical particles, Method of Reflections, Stokesian Dynamics

\section{Introduction}

The hydrodynamics of multi-particle suspensions in a Newtonian fluid matrix have been of interest for many years (see, for instance, [1]). Applications range from suspension rheology, through sedimentation problems to fluidized beds and beyond. Because colloidal particles are usually very small, there has been sustained interest in systems in which inertia is neglected. In this case the governing equations for the fluid matrix, the Stokes equations,

Email address: helen.wilson@ucl.ac.uk (Helen J. Wilson) 
are linear and quasi-static, which allows many mathematical tools to be used to simplify their numerical study.

In order to further simplify the analysis of such problems, and thus (in many cases) to permit the study of systems of larger numbers of particles, it is usual to consider the solid particles in the suspension as spheres, usually of identical size. Some progress has been made recently with ellipsoidal particles (for example, by $[2,3,4]$ ) and with spheres of different sizes $[5,6]$ but the bulk of the literature on viscous hard-sphere suspensions is concerned with systems of identical spheres.

There are five main families of methods of simulating such systems numerically.

The Finite Element Method (FEM) (see, for example, [7]) discretizes the whole fluid (and possibly the particle interiors as well), and as such is rather numerically intensive; however, it is easily extended to more general cases, including non-spherical particles, nonzero inertia, and other (non-Newtonian) constitutive equations for the fluid matrix.

Dissipative Particle Dynamics (DPD) discretizes space only on a square grid, regardless of the positions of the particles. As such, it is very fast but struggles to capture the lubrication interactions between close particles. These may be added by hand, at the expense of some of the computational speed and simplicity.

In the Boundary Element Method (BEM), used by [8, 9] and many others, the surface of each particle is discretized and the linearity of the Stokes equations used to reduce the problem to a set of linear equations involving the variables on the surface of the spheres. The inherent difficulty here occurs when two spheres are close together: then the inaccuracy of modelling of the sphere surfaces becomes important and the lubrication forces between two spheres may not be captured well. The method does not rely in any sense on the spherical shape of the particles, so other shapes can be captured without any difficulty.

Multipole methods (see, for instance, [10]) represent the geometry of the spheres exactly and are therefore restricted to spheres (or, with substantial effort, ellipsoids) and planes. The flow field is represented in terms of a resistance matrix (extracted from a truncated multipole expansion) relating the external forces and torques on all the spheres to their velocities and any background flow. The most popular method of this class is Stokesian Dynamics (SD), introduced by [11], in which the multipole expansion is truncated after the stresslets (force dipole) and the irreducible quadrupole. 
Finally, there are methods based on the Method of Reflections (MR) (described in, for example, [1]) in which the flow field outside each sphere is considered in the vicinity of another sphere, where the rigidity of the second sphere causes a perturbation flow field, which is then reflected onto all other spheres iteratively. These methods are only suitable for small numbers of spherical particles, and converge quickly only when the spheres are wellseparated. Indeed, some authors have found problems which do not appear to converge at all. However, if sufficient iterations are carried out and convergence does occur, these methods may be considered exact. In the current paper, we present a numerical scheme based on MR for three spheres, but with some improvements over the previous published studies in the field.

The paper is arranged as follows. In section 2 we describe our numerical method. In section 2.6 we discuss convergence issues and how problems may be avoided, and in section 3 validate our method against existing results, including a comparison with the BEM. Section 4 sees a quantitative comparison with the standard multipole method, Stokesian Dynamics: this is the first time the accuracy of SD has been quantified. Finally, in section 5 we demonstrate an example system having spheres of different sizes.

\section{Numerical method}

We present here a numerical solution technique for Stokes flow of a fluid surrounding solid spheres, based on the Method of Reflections [1], which exploits fully the spherical geometry of our particles. Although the results we present are primarily for three identical spheres, the method is capable of handling spheres of different radius with no difficulty.

The equations we solve are the incompressible Stokes equations:

$$
\boldsymbol{\nabla} \cdot \boldsymbol{u}=0 \quad \nabla p=\mu \nabla^{2} \boldsymbol{u}
$$

in which $\boldsymbol{u}$ is the fluid velocity and $p$ the pressure; $\mu$ is the fluid viscosity. We also wish to impose boundary conditions on the particles: either a velocity $\boldsymbol{U}$ and angular velocity $\boldsymbol{\Omega}$ for each particle, or an external force $\boldsymbol{F}$ and torque $\boldsymbol{T}$ acting on each particle.

We begin by constructing, for each particle, the flow field that satisfies the Stokes equations, and the boundary conditions on that particle, and decays in the far field. Then we proceed iteratively as follows: 


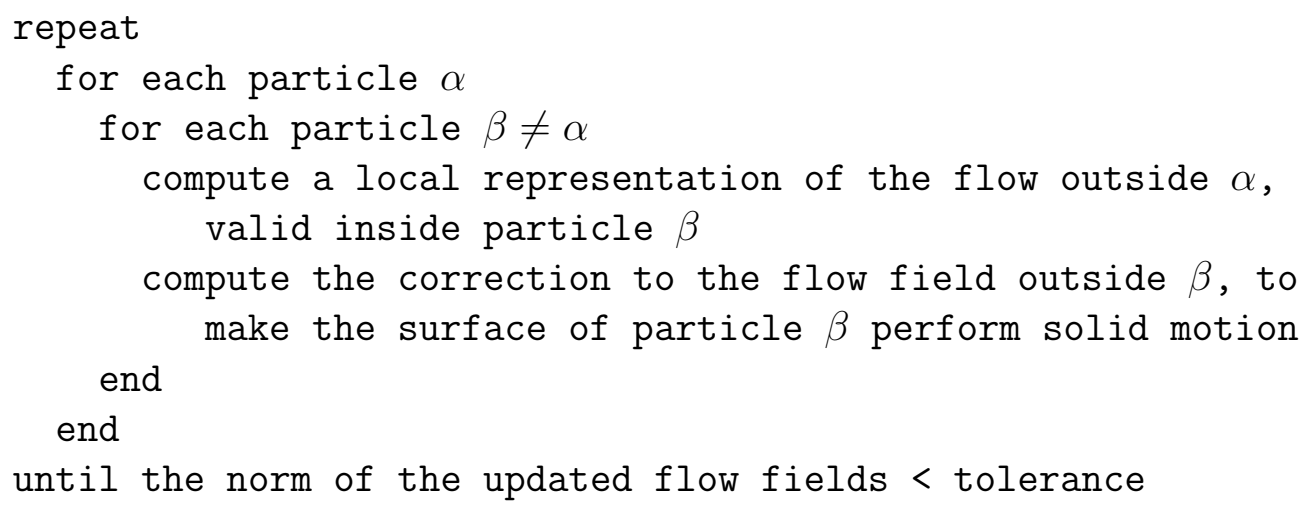

The two central calculations are described in sections 2.2 and 2.3 respectively.

To carry out this process, we write the general solution to the Stokes equations in spherical polar coordinates centred on a point $\boldsymbol{x}^{\alpha}$, using Lamb's solution (and writing $\boldsymbol{r}_{\alpha}=\boldsymbol{x}-\boldsymbol{x}^{\alpha}$ and $r_{\alpha}=\left|\boldsymbol{r}_{\alpha}\right|$ ):

$$
\begin{aligned}
\boldsymbol{u}= & \sum_{n=1}^{\infty} \sum_{m=-n}^{n}\left[A_{n m} \nabla\left(r_{\alpha}^{-n-1} Y_{n m}\left(\boldsymbol{r}_{\alpha}\right)\right)+B_{n m} \nabla \times\left(r_{\alpha}^{-n-1} \boldsymbol{r}_{\alpha} Y_{n m}\left(\boldsymbol{r}_{\alpha}\right)\right)\right. \\
- & \left.\frac{(n-2) C_{n m} r_{\alpha}^{2}}{2 n(2 n-1)} \nabla\left(r_{\alpha}^{-n-1} Y_{n m}\left(\boldsymbol{r}_{\alpha}\right)\right)+\frac{(n+1) C_{n m} r_{\alpha}^{-n-1}}{n(2 n-1)} \boldsymbol{r}_{\alpha} Y_{n m}\left(\boldsymbol{r}_{\alpha}\right)\right] \\
& +\sum_{n=0}^{\infty} \sum_{m=-n}^{n}\left[D_{n m} \nabla\left(r_{\alpha}^{n} Y_{n m}\left(\boldsymbol{r}_{\alpha}\right)\right)+E_{n m} \nabla \times\left(r_{\alpha}^{n} \boldsymbol{r}_{\alpha} Y_{n m}\left(\boldsymbol{r}_{\alpha}\right)\right)\right. \\
& \left.+\frac{(n+3) F_{n m} r_{\alpha}^{2}}{2(n+1)(2 n+3)} \nabla\left(r_{\alpha}^{n} Y_{n m}\left(\boldsymbol{r}_{\alpha}\right)\right)-\frac{n F_{n m} r_{\alpha}^{n}}{(n+1)(2 n+3)} \boldsymbol{r}_{\alpha} Y_{n m}\left(\boldsymbol{r}_{\alpha}\right)\right] \\
& p=\mu \sum_{n=1}^{\infty} \sum_{m=-n}^{n} C_{n m} r_{\alpha}^{-n-1} Y_{n m}\left(\boldsymbol{r}_{\alpha}\right)+\mu \sum_{n=0}^{\infty} \sum_{m=-n}^{n} F_{n m} r_{\alpha}^{n} Y_{n m}\left(\boldsymbol{r}_{\alpha}\right) .
\end{aligned}
$$

In general, the spherical harmonic of order $n$ is given by

$$
Y_{n m}(\boldsymbol{r})=(\operatorname{sgn}(m))^{m}\left[\frac{(2 n+1)(n-|m|) !}{4 \pi(n+|m|) !}\right]^{1 / 2} P_{n}^{|m|}(\cos \theta) \exp (\mathrm{i} m \phi),
$$

in which $\theta$ and $\phi$ are the usual spherical polar angles $(0 \leq \theta \leq \pi, 0 \leq \phi<2 \pi)$ and $P_{n}^{m}(x)$ are the associated Legendre polynomials, defined in the standard way from the Legendre polynomials $P_{n}(x)$ [12]:

$$
P_{n}^{m}(x)=(-1)^{m}\left(1-x^{2}\right)^{m / 2} \frac{\mathrm{d}^{m} P_{n}(x)}{\mathrm{d} x^{m}} .
$$


A flow field that decays at infinity has $D_{n m}=E_{n m}=F_{n m}=0$, so we can store such a field, valid outside a particle centred at $\boldsymbol{x}^{\alpha}$, simply as the set of coefficients $A_{n m}^{\alpha}, B_{n m}^{\alpha}$ and $C_{n m}^{\alpha}$.

For convenience, we also define here the constants

$$
h_{n}^{m}=\left[\frac{(2 n+1)(n-|m|) !}{4 \pi(n+|m|) !}\right]^{1 / 2} \quad g_{\nu n}^{m}=\frac{(n+\nu) !}{(\nu+|m|) !(n-|m|) !} .
$$

\subsection{Implementing initial conditions}

Suppose that particle $\alpha$, of radius $a_{\alpha}$, has associated with it polar coordinates $\left(r_{\alpha}, \theta, \phi\right)$ and also Cartesian coordinates $x=r_{\alpha} \sin \theta \cos \phi, y=$ $r_{\alpha} \sin \theta \sin \phi$ and $z=r_{\alpha} \cos \theta$. Then to impose a velocity $U_{x} \hat{\boldsymbol{x}}+U_{y} \hat{\boldsymbol{y}}+U_{z} \hat{\boldsymbol{z}}$ and angular velocity $\Omega_{x} \hat{\boldsymbol{x}}+\Omega_{y} \hat{\boldsymbol{y}}+\Omega_{z} \hat{\boldsymbol{z}}$ on particle $\alpha$, the initial flow field we require, valid outside that particle $\alpha$, is defined by the constants

$$
\begin{aligned}
& A_{1,-1}=\frac{\left[U_{x}+\mathrm{i} U_{y}\right] a_{\alpha}^{3}}{8 h_{1}^{1}} \quad A_{10}=\frac{U_{z} a_{\alpha}^{3}}{4 h_{1}^{0}} \quad A_{11}=\frac{\left[-U_{x}+\mathrm{i} U_{y}\right] a_{\alpha}^{3}}{8 h_{1}^{1}} \\
& B_{1,-1}=\frac{\left[\Omega_{x}+\mathrm{i} \Omega_{y}\right] a_{\alpha}^{3}}{2 h_{1}^{1}} \quad B_{10}=\frac{\Omega_{z} a_{\alpha}^{3}}{h_{1}^{0}} \quad B_{11}=\frac{\left[-\Omega_{x}+\mathrm{i} \Omega_{y}\right] a_{\alpha}^{3}}{2 h_{1}^{1}} \\
& C_{1,-1}=\frac{3\left[U_{x}+\mathrm{i} U_{y}\right] a_{\alpha}}{4 h_{1}^{1}} \quad C_{10}=\frac{3 U_{z} a_{\alpha}}{2 h_{1}^{0}} \quad C_{11}=\frac{3\left[-U_{x}+\mathrm{i} U_{y}\right] a_{\alpha}}{4 h_{1}^{1}}
\end{aligned}
$$

with all other coefficients being zero. Similarly, a force $\boldsymbol{F}$ may be imposed with the same coefficients as for a velocity $\boldsymbol{U}=\boldsymbol{F} / 6 \pi \mu a_{\alpha}$, and a torque $\boldsymbol{T}$ with the same coefficients as an angular velocity $\boldsymbol{\Omega}=\boldsymbol{T} / 8 \pi \mu a_{\alpha}^{3}$.

\subsection{Expressing a field valid outside one sphere as one valid inside another}

We now consider the process required to express a field valid outside particle $\alpha$ (expressed as a set of coefficients $A_{n m}^{\alpha}, B_{n m}^{\alpha}$ and $C_{n m}^{\alpha}$ ) as an expansion valid inside particle $\beta$ : that is, coefficients $D_{n m}^{\beta}, E_{n m}^{\beta}$ and $F_{n m}^{\beta}$.

We begin by rotating the flow field into a new set of coordinates, still centred on $\boldsymbol{x}^{\alpha}$, whose $z$-axis is aligned with the line joining $\boldsymbol{x}^{\alpha}$ and $\boldsymbol{x}^{\beta}$. This is achieved using a rotation formula derived by [13]. For two spherical coordinate systems $(r, \theta, \phi)$ and $\left(r, \theta^{\prime}, \phi^{\prime}\right)$ whose axes $\phi=\pi / 2$ are aligned, and whose $\theta=0$ axes differ by an angle $\theta_{0}$, we have

$$
Y_{l m}(\theta, \phi)=\sum_{t=-m}^{m} \mathrm{i}^{t-m} Q_{m t}^{l}\left(\theta_{0}\right) Y_{l t}\left(\theta^{\prime}, \phi^{\prime}\right)
$$


in which the rotation matrix is calculated through the recurrence relations

$$
\begin{gathered}
Q_{l t}^{l}=\sqrt{g_{l l}^{t}}\left(\frac{1-\cos \theta_{0}}{2}\right)^{(l-t) / 2}\left(\frac{1+\cos \theta_{0}}{2}\right)^{(l+t) / 2} \\
\frac{Q_{m-1, t}^{l}}{\sqrt{g_{l l}^{m-1}}}+\frac{2\left(t-m \cos \theta_{0}\right)}{(l+m) \sin \theta_{0}} \frac{Q_{m t}^{l}}{\sqrt{g_{l l}^{m}}}+\frac{(l-m)}{(l+m)} \frac{Q_{m+1, t}^{l}}{\sqrt{g_{l l}^{m+1}}}=0 .
\end{gathered}
$$

Because we are only considering three spheres, we can fix the axes $\phi=0$ to lie within the plane of the spheres' centres, which means the axes $\phi=\pi / 2$ will always lie perpendicular to that plane. In practice, we arrange the coordinate systems on the three spheres to minimise these rotations: so spheres 1 and 2 have coordinate systems whose $z$-axes are parallel to the line joining their centres, and sphere 3's coordinate system has its $z$-axis pointing towards one of the other two spheres. This leaves us with only three matrices $Q\left(\theta_{0}\right)$ to calculate, and these are stored in advance.

We now have a set of coefficients $\{A, B, C\}_{n m}^{\alpha}$ describing a flow field valid outside particle $\alpha$, in a coordinate system whose $z$-axis points towards the centre of particle $\beta$. To convert this into a flow field valid inside particle $\beta$, we use the generalized addition theorem [14], valid for two coordinate systems with their $\theta=0$ axes aligned:

$$
\frac{Y_{n m}\left(\boldsymbol{r}_{\alpha}\right)}{r_{\alpha}^{n+1}}=\sum_{\nu=|m|}^{\infty} \frac{h_{n}^{m}}{h_{\nu}^{m}} \frac{(-1)^{n+m}( \pm 1)^{n+\nu} g_{\nu n}^{m}}{r_{\alpha \beta}^{n+\nu+1}} r^{\nu} Y_{\nu m}\left(\boldsymbol{r}_{\beta}\right)
$$

in which $r_{\alpha \beta}=\left|\boldsymbol{x}^{\beta}-\boldsymbol{x}^{\alpha}\right|$. The appearance of the term $r_{\alpha \beta}^{n+\nu+1}$ reduces the magnitude of the coefficients in the new coordinate system relative to those in the old coordinates: this is what drives the convergence of the numerical method. The coefficients in the new coordinate system become

$$
\begin{aligned}
& D_{\nu m}^{\beta}=\sum_{n=|m|}^{\infty} \frac{h_{n}^{m}}{h_{\nu}^{m}} \frac{(-1)^{n+m}( \pm 1)^{n+\nu}}{r_{\alpha \beta}^{n+\nu+1}}\left[g_{\nu n}^{m} A_{n m}^{\alpha} \mp \mathrm{i} m \frac{g_{\nu n}^{m}}{\nu} r_{\alpha \beta} B_{n m}^{\alpha}\right. \\
& \left.+\left\{g_{\nu-1, n}^{m} \frac{(\nu-|m|)((\nu-1)(n-2)-(n+1))}{\nu(2 \nu-1)}-g_{\nu n}^{m} \frac{(n-2)}{2}\right\} \frac{r_{\alpha \beta}^{2} C_{n m}^{\alpha}}{n(2 n-1)}\right] \\
& E_{\nu m}^{\beta}=\sum_{n=|m|}^{\infty} g_{\nu n}^{m} \frac{h_{n}^{m}}{h_{\nu}^{m}} \frac{(-1)^{n+m}( \pm 1)^{\nu+n}}{r_{\alpha \beta}^{n+\nu+1}}\left[-\frac{n}{(\nu+1)} B_{n m}^{\alpha} \mp \frac{\mathrm{i} m r_{\alpha \beta} C_{n m}^{\alpha}}{n \nu(\nu+1)}\right]
\end{aligned}
$$




$$
F_{\nu m}^{\beta}=\sum_{n=|m|}^{\infty} \frac{h_{n}^{m}}{h_{\nu}^{m}} \frac{(-1)^{n+m}( \pm 1)^{n+\nu} g_{\nu n}^{m}}{r_{\alpha \beta}^{n+\nu+1}} C_{n m}^{\alpha} .
$$

with each \pm term being + if the centre of particle $\beta$ is at $\theta=\pi$ in the axes of particle $\alpha,-$ if it is at $\theta=0$.

The new set of coefficients $\{D, E, F\}_{n m}^{\beta}$ may also need rotating again into the standard coordinate system of particle $\beta$ before we continue; this is carried out just as it was at the beginning of this section.

\subsection{Additional flow field to return particle $\beta$ to solid-body motion}

We now have a flow field $\boldsymbol{u}_{\text {inside }}$ valid inside sphere $\beta$, and need to calculate what additional flow field we need to ensure that the particle only undergoes solid motion. Essentially, we wish to find a flow field $\boldsymbol{u}_{\text {outside }}$, valid outside particle $\beta$, such that on the particle surface,

$$
\boldsymbol{u}_{\text {inside }}+\boldsymbol{u}_{\text {outside }}=\boldsymbol{U}+\boldsymbol{\Omega} \times \boldsymbol{r}_{\beta}
$$

for some constant vectors $\boldsymbol{U}$ and $\boldsymbol{\Omega}$. This requirement gives, for $n>1$,

$$
\begin{aligned}
& A_{n m}^{\beta}=-\frac{n a_{\beta}^{2 n+1}}{4(n+1)(2 n+3)}\left[2(2 n-1)(2 n+3) D_{n m}^{\beta}+(2 n+1) a_{\beta}^{2} F_{n m}^{\beta}\right], \\
& B_{n m}^{\beta}=-a_{\beta}^{2 n+1} E_{n m}^{\beta}, \\
& C_{n m}^{\beta}=-\frac{n(2 n-1) a_{\beta}^{2 n-1}}{2(n+1)}\left[2(2 n+1) D_{n m}^{\beta}+a_{\beta}^{2} F_{n m}^{\beta}\right] .
\end{aligned}
$$

The terms at $n=1$ may be different as they include contributions from $\boldsymbol{U}$ and $\boldsymbol{\Omega}$. Here there are two cases: if we are considering a resistance problem, then we have already imposed our velocity and angular velocity, and the extra velocities $\boldsymbol{U}$ and $\boldsymbol{\Omega}$ added at this stage must be zero. Then the formulae of equations (18-20) are also valid at $n=1$.

If we are considering a mobility problem, however, the fixed quantity is the force and torque imposed on each particle:

$$
\begin{aligned}
& \boldsymbol{F}_{\text {imposed }}=-4 \pi \mu \sum_{m=-1}^{1} C_{1 m} \nabla\left(r Y_{1 m}(\boldsymbol{r})\right), \\
& \boldsymbol{T}_{\text {imposed }}=-8 \pi \mu \sum_{m=-1}^{1} B_{1 m} \nabla\left(r Y_{1 m}(\boldsymbol{r})\right) .
\end{aligned}
$$


Thus we cannot add to $B_{1 m}$ and $C_{1 m}$ with our perturbation field, and instead we just have the condition

$$
A_{1 m}^{\beta}=-\frac{F_{1 m}^{\beta} a_{\beta}^{5}}{30}
$$

\subsection{Finishing the calculation}

When this process has been repeated, for each pair of particles, sufficient times that the additional coefficients are negligibly small, we have three flow fields (one valid outside each particle) each of which describes the flow everywhere in the fluid. It only remains to extract either the forces and torques imposed on the particle (for the resistance question) or their velocities and angular velocities (for the mobility question).

The resistance problem requires only the values $B_{1 m}$ and $C_{1 m}$, as we saw from equations (21-22). The force and torque, in the natural coordinate system, are then given by

$$
\begin{aligned}
& F_{x}=4 \pi \mu h_{1}^{1}\left(C_{11}-C_{1,-1}\right), F_{y}=4 \pi \mu h_{1}^{1} \mathrm{i}\left(C_{1,-1}+C_{11}\right), F_{z}=-4 \pi \mu h_{1}^{0} C_{10}, \\
& T_{x}=8 \pi \mu h_{1}^{1}\left(B_{11}-B_{1,-1}\right), T_{y}=8 \pi \mu h_{1}^{1} \mathrm{i}\left(B_{1,-1}+B_{11}\right), T_{z}=-8 \pi \mu h_{1}^{0} B_{10}
\end{aligned}
$$

(in which, from equation (6), $h_{1}^{0}=[3 / 4 \pi]^{1 / 2}$ and $h_{1}^{1}=[3 / 8 \pi]^{1 / 2}$ ).

In the mobility problem, the values of $C_{1 m}$ and $B_{1 m}$ are unchanged from their initial conditions, and instead we extract the velocity and angular velocity of each particle. If we define the velocities as

$$
\boldsymbol{U}=\sum_{m=-1}^{1} U_{m} \nabla\left(r Y_{1 m}(\boldsymbol{r})\right) \quad \boldsymbol{\Omega}=\sum_{m=-1}^{1} \Omega_{m} \nabla\left(r Y_{1 m}(\boldsymbol{r})\right)
$$

then the coefficients are given by

$$
U_{m}=\frac{3 C_{1 m}}{2 a}-\frac{5 A_{1 m}}{a^{3}}+D_{1 m} \quad \Omega_{m}=E_{1 m}+\frac{B_{1 m}}{a^{3}}
$$

and the velocities,

$$
\begin{gathered}
U_{x}=h_{1}^{1}\left(U_{-1}-U_{1}\right), \quad U_{y}=-h_{1}^{1} \mathrm{i}\left(U_{-1}+U_{1}\right), \quad U_{z}=h_{1}^{0} U_{0}, \\
\Omega_{x}=h_{1}^{1}\left(\Omega_{-1}-\Omega_{1}\right), \quad \Omega_{y}=-h_{1}^{1} i\left(\Omega_{-1}+\Omega_{1}\right), \quad \Omega_{z}=h_{1}^{0} \Omega_{0} .
\end{gathered}
$$




\subsection{Numerical parameters}

The only question that remains is where to terminate the infinite sums: that is, what value of $N$ to use in the approximations (for any $\mathcal{F}$ )

$$
\sum_{n=1}^{\infty} \sum_{m=-n}^{n} \mathcal{F}\left\{Y_{n m}\left(\boldsymbol{x}-\boldsymbol{x}^{\alpha}\right)\right\} \approx \sum_{n=1}^{N} \sum_{m=-n}^{n} \mathcal{F}\left\{Y_{n m}\left(\boldsymbol{x}-\boldsymbol{x}^{\alpha}\right)\right\}
$$

If we are to iterate to a global error of $\epsilon$, say, then how should we select $N$ ? Answering an equivalent question, [13] argued that in terms of a dimensionless particle separation defined as

$$
\zeta_{\alpha \beta}=\frac{2 r_{\alpha \beta}}{\left(a_{\alpha}+a_{\beta}\right)}
$$

the key quantity is $q_{\alpha \beta}<1$ which satisfies $\zeta_{\alpha \beta}=q_{\alpha \beta}+q_{\alpha \beta}^{-1}$. He showed that the error term caused by neglecting the $N$ th term of the expansion for the flow around particle $\alpha$ caused by particle $\beta$ is of magnitude

$$
N^{-1 / 2} q_{\alpha \beta}^{N}
$$

The decay of this term is dominated by the exponential term $q^{N}$, so in order to achieve $q_{\alpha \beta}^{N}<\epsilon$ we set

$$
N_{\alpha \beta}=\left\lceil\frac{\log \epsilon}{\log q_{\alpha \beta}}\right\rceil
$$

For all particles, we keep terms up to the largest $N_{\alpha \beta}$ in the whole system; but when storing the rotation matrices (which are calculated in advance) we use the value of $N_{\alpha \beta}$ appropriate to the pair of particles in question.

We terminate the calculation (that is, stop the iterations) when the most recent iteration has added a contribution of magnitude less than $0.01 \epsilon$ to any of the forces, torques, velocities and angular velocities.

The largest matrices stored are the rotation matrices (3 for the case of three spheres), which each contain approximately $4 N_{\alpha \beta}^{3}$ elements. Given the various occasions where these are passed as parameters, and the 32-bit limit of addresses in many compilers, these large matrices effectively limit the maximal value of $N_{\alpha \beta}$ we can use to 150 .

In terms of computational time, the most complex operation is also the rotation stage: of order $N_{\alpha \beta}^{3}$ per rotation. For the case of $M$ spheres, there will be $2 M(M-2)$ rotations for each iteration. 
As an illustration, we look at the case of three equal spheres arranged in an equilateral triangle, and fix $\epsilon=10^{-5}$. If the surface gap is $0.1 a$, then $N_{\alpha \beta}=37$ and the calculation takes 55 iterations to converge. This takes about $25 \mathrm{~s}$ on a standard laptop ${ }^{1}$ : so each iteration takes roughly 0.45 seconds. With a surface gap of $0.01 a, N_{\alpha \beta}=116$ and the calculation requires around 1000 iterations. On the same laptop this took around 3 hours: so each iteration is now taking $10.8 \mathrm{~s}$. In each case the time taken per iteration is of the order $10^{-5} N_{\alpha \beta}^{3} \mathrm{~s}$.

\subsection{Convergence of the method}

Our program is formulated so that we can consider either the resistance problem (impose velocities and angular velocities on the particles, and calculate the required forces and torques) or the mobility problem (impose forces and torques, and calculate the resultant velocities). Clearly in a philosophical sense these two problems are equivalent: but numerically we have found empirically that their convergence behaves very differently. For well-separated spheres there are no convergence issues in either problem, but when the spheres are close together the resistance problem is found to converge much worse than the mobility problem.

As an example of this (revisited in much more detail in §3), we considered a system of three identical spheres arranged in an equilateral triangle, moving out of the plane of their centres. The resistance problem converges in this case only when the triangle side is at least 2.3 times the particle radius; the mobility problem seems to converge unconditionally (though of course there are memory issues for very close spheres below a separation of 2.05 radius).

It is not entirely surprising that the mobility problem should behave better than the resistance problem: for many flow arrangements, the resistance functions are singular as particles approach contact, whereas the corresponding mobility functions tend to zero. Indeed, [15] showed that for two-sphere problems (using $s$ as the dimensionless separation, with $s=2$ at contact), for many resistance functions the series of contributions decay as the terms of the Taylor series for $(1+2 / s)^{-1}$ and $(\ln (1+2 / s))^{-1}$, i.e. rather slowly for $s$ close to 2 , whereas the mobility functions were at worst regular functions with singularities in their derivatives at contact. However, in a system such as the one described above, where there is no singularity and all forces,

\footnotetext{
${ }^{1}$ Intel Core 2 Duo $1.3 \mathrm{GHz}$ laptop with $2 \mathrm{~GB}$ of RAM
} 
torques and velocities remain finite, we still retain the strong improvement in performance by considering the mobility problem rather than the resistance problem. The reason for this ${ }^{2}$ is that in the zero-th order (the single-sphere) term, one already accounts for all the force and torque on the sphere. So each subsequent reflection starts with a stresslet term as the leading term in the multipole expansion for the reflection field.

Of course, the whole problem is linear, because of the linearity of the Stokes equations, and can be thought of as a linear system whose unknowns are the set of coefficient variables $\{A, B, C\}$. Thus we are talking about the convergence of a linear system, with a sparse matrix of a particular structure. Our iterative scheme is similar to a Gauss-Seidel iteration, with a particular ordering of the unknowns; and the mobility and resistance formulations may be considered as a form of preconditioning. Perhaps this insight could open the possibility of other forms of preconditioning which are even more stable than the mobility formulation.

The results in the rest of the paper are all calculated using the mobility formulation of the problem.

\subsection{Stability of the calculations}

In this program we use relatively high degrees (up to 150) of spherical harmonics. Most recurrence relations for computing these harmonics are subject to cancellation and mild instabilities, and have problems for high degrees and orders. However, the stability of the recurrence relation given by equations (11-12) was discussed in Appendix B of [13] (in which it is equation C6). They used degrees up to 550 without any loss of accuracy.

\section{Validation of the method}

We validated the program against all the two-sphere results available in the literature, many of which are tabulated in [16], but there are only two test cases available for three spheres.

\subsection{Triangle of spheres}

The first three-sphere test case is the setup discussed briefly in section 2.6, which was first studied by [17]. He used a MR method, but without the

\footnotetext{
${ }^{2}$ Explanation by Sangtae Kim; private correspondence
} 
rotations included in our code, and he restricted himself to the resistance problem, which we have seen has adverse effects on convergence. We can easily reconstruct resistance results and in this section we carry out a detailed comparison with his data. The same system was studied using a BEM by [8], whose results for very close spheres illustrate the difficulty of finding accurate solutions when lubrication interactions are involved.

Consider an equilateral triangle of three equal spheres, each of radius $a$, with centres separated by a distance as, moving in the direction perpendicular to their plane of centres. The standard resistance problem for this setup would be to impose the velocity (and zero angular velocity) and calculate the required force and torque. This was the problem addressed by Kim, who published only the force results (not those for torque). He also had convergence problems for $s \leq 2.16$, which were slightly improved by some clever accelerated series. In figure 1 we reproduce part of his figure 1, showing his numerical results for the drag. The crosses are from his original numerical method, the asterisks from the accelerated series. The results from figure 10 of [8], which used the Boundary Element Method, are also plotted on figure 1 .

To reproduce this information using the mobility code requires two separate problems to be studied. In the first, we apply a unit force (out of the plane) to each sphere. This results in an out-of-plane velocity but also in rotations of each sphere about an axis which is parallel to the line joining the centres of the other two spheres. We denote these values by $M_{U F}$ and $M_{\Omega F}$ respectively. Second, we impose a symmetrical set of unit torques to the spheres, in the same directions that the angular velocities occurred in the previous problem. This results in a new set of angular velocities about those same axes, $M_{\Omega T}$, and a new set of equal velocities out of the plane, $M_{U T}$. It can be proved that $M_{U T}=M_{\Omega F}$ using the reciprocal theorem.

Given this information, we can formulate the matrix systems:

$$
\begin{gathered}
\left(\begin{array}{c}
U \\
\Omega
\end{array}\right)=\underline{\underline{M}}\left(\begin{array}{l}
F \\
T
\end{array}\right)=\left(\begin{array}{ll}
M_{U F} & M_{\Omega F} \\
M_{U T} & M_{\Omega T}
\end{array}\right)\left(\begin{array}{c}
F \\
T
\end{array}\right) \\
\left(\begin{array}{c}
F \\
T
\end{array}\right)=\underline{\underline{R}}\left(\begin{array}{l}
U \\
\Omega
\end{array}\right)=\left(\begin{array}{ll}
R_{F U} & R_{F \Omega} \\
R_{T U} & R_{T \Omega}
\end{array}\right)\left(\begin{array}{c}
U \\
\Omega
\end{array}\right)
\end{gathered}
$$

so that $\underline{\underline{R}}=\underline{\underline{M}}^{-1}$ and Kim's results for $R_{F U}$ can be reconstructed as

$$
R_{F U}=\frac{M_{\Omega T}}{M_{U F} M_{\Omega T}-M_{\Omega F} M_{U T}}=\frac{M_{\Omega T}}{M_{U F} M_{\Omega T}-M_{U T}^{2}} .
$$




$\begin{array}{cccccc}s & M_{U F} & M_{\Omega F} & M_{\Omega T} & M_{U T} & R_{F U} \\ 3.00 & 1.53416156 & 0.140187608 & 0.771951396 & 0.140187608 & 0.662821 \\ 2.90 & 1.55461831 & 0.149081378 & 0.772261325 & 0.149081378 & 0.655377 \\ 2.80 & 1.57664602 & 0.158630515 & 0.771969753 & 0.158630515 & 0.647648 \\ 2.70 & 1.60039150 & 0.168801210 & 0.770692987 & 0.168801210 & 0.639623 \\ 2.60 & 1.62599574 & 0.179481457 & 0.767809702 & 0.179481457 & 0.631297 \\ 2.50 & 1.65356957 & 0.190411103 & 0.762285310 & 0.190411103 & 0.622662 \\ 2.40 & 1.68314045 & 0.201041187 & 0.752325593 & 0.201041187 & 0.613716 \\ 2.30 & 1.71452988 & 0.210224103 & 0.734617762 & 0.210224103 & 0.604460 \\ 2.25 & 1.73072508 & 0.213577673 & 0.720980855 & 0.213577673 & 0.599716 \\ 2.20 & 1.74703222 & 0.215412007 & 0.702342997 & 0.215412007 & 0.594897 \\ 2.15 & 1.76311004 & 0.214756269 & 0.676197489 & 0.214756269 & 0.590004 \\ 2.10 & 1.77826951 & 0.209731929 & 0.637667933 & 0.209731929 & 0.585039 \\ 2.05 & 1.79070892 & 0.195478184 & 0.573859459 & 0.195478184 & 0.580005 \\ 2.01 & 1.79223228 & 0.159607490 & 0.455596646 & 0.159607101 & 0.575932\end{array}$

Table 1: Mobility and resistance results for a system of three equidistant spheres moving out of their plane.

In table 1 we give the results for the mobility calculations for a range of values of $s$. In all cases except $s=2.01$, the results have converged to a global error of $10^{-13}$; at $s=2.01$ memory constraints on the computer available limited the number of terms which could be kept, so a global error of $3 \times 10^{-6}$ was used.

We investigate the limit of touching spheres, $R_{F U}^{T}$. Extrapolation of the last six evenly spaced data points (the range $s=2.05-2.3$ ) gives a linear fit of

$$
R_{F U}=0.5752+0.0978(s-2) .
$$

The $90 \%$ confidence interval on the slope here is [0.0964,0.0993]; that on the intercept, $[0.5745,0.5759]$. The resultant estimate for the drag coefficient for a triangle of touching spheres is $R_{F U}^{T}=0.5752$. For comparison, Tran-Cong with their Boundary Element Method had a value of 0.583; Kim (extrapolating from the separations at which his program converged) predicted 0.585; and the only experimental data point available (from [18]) has $R_{F U}^{T}=0.574$. We can see that the current method produces a value much closer to that observed in experiments, than any of the previous attempts.

In figure 1 it is interesting to note that, while the BEM of [8] behaves better than the Method of Reflections of [17] for small separations (as one 


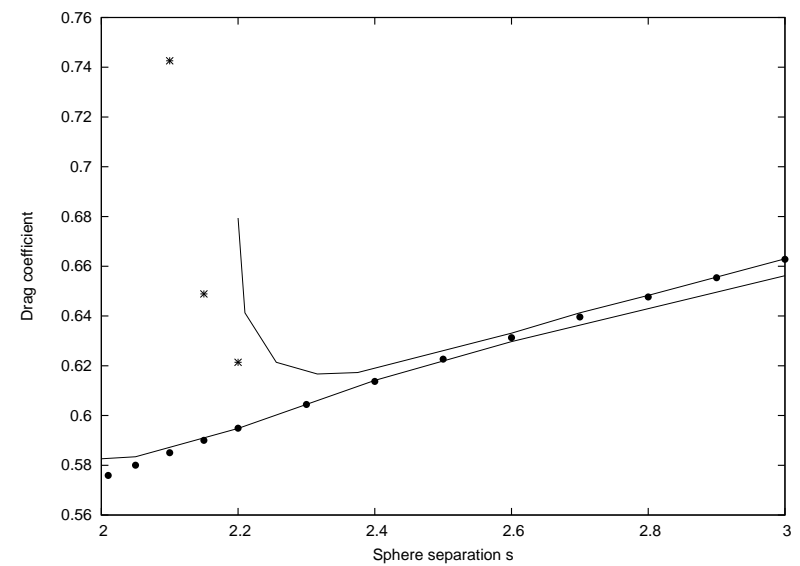

Figure 1: Drag coefficient for three identical spheres arranged with centres forming an equilateral triangle. The spheres each have radius $a$, the triangle has side $a s$, and the spheres are moving perpendicular to their plane of centres. The upper solid line is part of figure 1 from [17], showing his raw results, and the asterisks $*$ are his results after using accelerated series. The lower solid line is the numerical results from figure 10 of [8]. The circles • are our numerical results (shown explicitly as $R_{F U}$ in table 1). The linear extrapolation of our results to touching spheres $(s=2)$ gives a value for the drag coefficient at contact of 0.575 . 
would expect), for sphere separations greater than 2.6a the BEM results lose accuracy. The reason for this is not clear; the discretisation of the sphere surfaces must be the root cause of the inaccuracy, but why should it have a more marked effect at this separation than when the spheres are close together?

\subsection{Spheres in a line}

The second test case is also taken from [8]. Three spheres of radius $a$ are arranged equispaced on a line, with centre-to-centre separations as, and allowed to sediment (under a force which would produce unit velocity on an isolated sphere) perpendicular to that line. The two outer spheres fall at equal speeds $U_{1}$ and the centre sphere at speed $U_{0}$; and the outer spheres rotate at angular velocity $\Omega$.

Results are available from [8] for touching spheres $s=2$, and for separations of $s=2.05,2.1,2.4$, and 3 ; the same system at a separation $s=4$ (for 3 spheres and also larger numbers) was studied in figure 6 of [19] using a collocation method. The velocity values in the literature are all reported as a dimensionless drag $D_{i}=1 / U_{i}$, so we follow that convention. The angular velocities in [8] are made dimensionless using $U_{1} / a$; we follow the convention of [19] instead, and make them dimensionless relative to $U_{s} / a$, where $U_{s}$ is the Stokes settling velocity of an isolated sphere. These literature results have been read directly from figures with a ruler, and are thus only reproduced to three decimal places. In table 2 we report our velocities (converged to a global error of $10^{-8}$ ) along with those from the literature. We ignore the case of touching spheres, as we cannot reproduce it. The velocity (or drag) for all the spheres is reproduced to within graphical accuracy in all cases; the worst calculation is the angular velocity at a separation of $s=2.05$, and even there the relative error is only $1.8 \%$, which is within the magnitude of errors we observed in section 3.1 with the BEM of [8].

\section{Stokesian Dynamics: A Quantitative Study}

In this section we compare the numerical method of Stokesian Dynamics, SD [11], in its original form for a finite set of identical spheres in an unbounded fluid, against our exact results for three spheres. It should be borne in mind that the current method is not a candidate to replace SD: it is far too numerically intensive. Nonetheless, it is interesting to see what errors are inherent in the SD formulation. 


\begin{tabular}{ccccccc} 
& \multicolumn{3}{c}{ Literature } & \multicolumn{3}{c}{ Current Method } \\
$s$ & $D_{0}$ & $D_{1}$ & $\Omega$ & $D_{0}$ & $D_{1}$ & $\Omega$ \\
4.00 & 0.720 & 0.777 & 0.058 & 0.7194 & 0.7755 & 0.0586 \\
3.00 & 0.651 & 0.717 & 0.105 & 0.6522 & 0.7177 & 0.1036 \\
2.40 & 0.595 & 0.666 & 0.159 & 0.5946 & 0.6663 & 0.1578 \\
2.10 & 0.562 & 0.634 & 0.192 & 0.5610 & 0.6337 & 0.1919 \\
2.05 & 0.557 & 0.629 & 0.197 & 0.5561 & 0.6277 & 0.1934
\end{tabular}

Table 2: The dimensionless drag $D_{0}$ (centre sphere) and $D_{1}$ (outer spheres) and angular velocities $\Omega$ for three collinear spheres of radius $a$ equispaced at a distance as, sedimenting perpendicular to their line of centres. In the limit $s \rightarrow \infty, D_{i} \rightarrow 1$ and $\Omega \rightarrow 0$. The literature values for $s \leq 3$ are taken from figures 12 and 13 of [8], those for $s=4$ from figure 6 of [19].

Stokesian Dynamics is based on the multipole expansion. Far-field interactions are captured through a grand mobility matrix of two-body far-field interactions, which is inverted to give many-body effects such as screening. Then close-particle interactions are included by the addition of exact (tabulated) two-body resistance functions (and then a correction to avoid doublecounting). As such, it is designed to be accurate for well-separated particles, and lubrication forces between close particles with relative motion are also captured accurately. In order to assess the systematic errors inherent in the SD approximation, we consider several problems based on three spheres, which highlight the strengths and weaknesses of SD.

\subsection{Test case: Triangle moving out of plane}

Our first geometry is similar to that discussed in section 3.1: a system of three identical spheres of radius $a$ arranged in an equilateral triangle of side as. We apply a force $6 \pi \mu a$ to each sphere, acting perpendicular to the plane of the sphere centres. Since Stokesian Dynamics is formulated for dynamic simulation, it naturally considers the mobility problem rather than the resistance form, so we allow the spheres to rotate freely. To fully reproduce the data of [17] and [8] we would need to constrain the spheres not to rotate, or carry out two separate calculations as in section 3.1; since we have already validated our code against those results, here we simply carry out the mobility calculation and compare the resultant velocities and angular velocities. In figure 2 we plot the sphere velocities and the magnitude of the angular velocities against the dimensionless centre-to-centre separation $s$. 


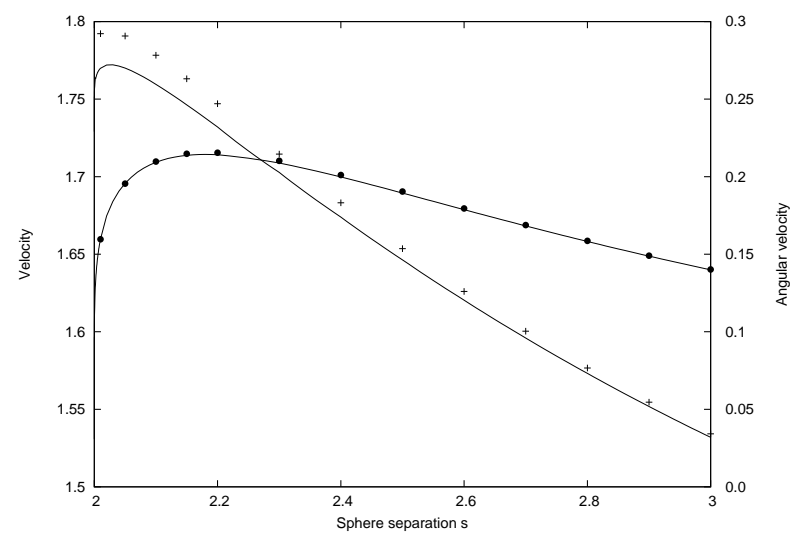

Figure 2: Velocities $(+)$ and angular velocities $(\bullet)$ for an equilateral triangle of spheres forced perpendicular to their plane of centres. The curves are from Stokesian Dynamics and the points from the current method. The angular velocities are captured by SD more accurately than the velocities, but neither case produces a relative error of more than around $1 \%$.

As we can see, the SD results agree with our results (to within graphical accuracy) for well-separated spheres; but as the spheres become close there is a systematic error in SD. The sphere velocities are underpredicted by SD, with the maximum relative error being for the closest spheres, where the velocities predicted by SD drop off sharply. These are not large errors: at a separation of 2.01, the true velocity is 1.792 , whereas SD predicts a velocity of 1.770 ; this is a relative error of only $1.2 \%$. The angular velocities are predicted to within graphical accuracy at all separations.

\subsection{Test case: Triangle with motion within the plane}

In this section we consider a problem which has only one (reflective) symmetry, and in which true lubrication forces occur, through the approach of close spheres. The spheres (each of radius $a$ ) are arranged in an equilateral triangle of side $s$ as before. A force of magnitude $6 \pi \mu a$ is applied to sphere 1 , acting towards the centroid of the triangle, and spheres 2 and 3 are force-free and torque-free. We denote the resultant velocity of sphere 1 as $U_{1}$, and the velocities of spheres 2 and 3 in the same direction as $U_{2}$. Spheres 2 and 3 also move apart with equal and opposite velocities $U_{3}$, and rotate with equal and opposite angular velocities of magnitude $a \Omega$. We show these velocities in table 3. In all cases our results may be considered exact (converged to a 


\begin{tabular}{|c|c|c|c|c|c|c|c|c|}
\hline \multirow[b]{2}{*}{$s$} & \multicolumn{4}{|c|}{ Current Method } & \multicolumn{4}{|c|}{$\mathrm{SD}$} \\
\hline & $U_{1}$ & $U_{2}$ & $U_{3}$ & $\Omega$ & $U_{1}$ & $U_{2}$ & $U_{3}$ & $\Omega$ \\
\hline 2.01 & 0.65528 & 0.63461 & 0.00498 & 0.037336 & 0.64739 & 0.62691 & 0.00451 & 0.034339 \\
\hline 2.10 & 0.73857 & 0.59718 & 0.03517 & 0.052035 & 0.73126 & 0.58784 & 0.02570 & 0.051414 \\
\hline 2.50 & 0.87765 & 0.49545 & 0.07393 & 0.045466 & 0.87482 & 0.48829 & 0.05853 & 0.045446 \\
\hline 3.00 & 0.93905 & 0.41694 & 0.07824 & 0.035022 & 0.93806 & 0.41356 & 0.06970 & 0.034843 \\
\hline 4.00 & 0.97964 & 0.31859 & 0.06925 & 0.021634 & 0.97945 & 0.31774 & 0.06639 & 0.021581 \\
\hline 6.00 & 0.99581 & 0.21586 & 0.05078 & 0.010159 & 0.99579 & 0.21575 & 0.05019 & 0.010153 \\
\hline
\end{tabular}

Table 3: Velocities and angular velocities for a triangle of spheres of radius $a$, arranged in an equilateral triangle of side $a s$, one of which is driven towards the triangle centroid with a force $6 \pi \mu a$. It has resultant velocity $U_{1} ; U_{2}$ is the induced velocity in the other two spheres in the same direction, and $\pm U_{3}$ their velocity perpendicular to the forcing. They rotate with angular velocity $\pm a \Omega$. Results are shown for our method and for Stokesian Dynamics (SD).

global error of at most $10^{-7}$ ).

The quantity $U_{3}$, which is the antisymmetric velocity of the unforced spheres. is the most susceptible to errors, both in absolute and relative terms. It is underrepresented by SD at all separations, with the worst errors occurring at intermediate separations. At $s=2.01$ the relative error is $9 \%$; at 2.1 it is $27 \%$ and even at 2.5 , with relatively well-spaced spheres, the relative error is $21 \%$. Thereafter it decreases with increasing $s$, and for very well-separated spheres at $s=6$ the relative error is only around $1 \%$.

\subsection{Test case: Spheres in a line}

In this final test case, we arrange our three spheres equispaced in a line (with centre-to-centre separation $a s$ ), and apply a force $6 \pi \mu a$ to the middle sphere, acting towards one of the others. The two outer spheres have equal velocities $U_{1}$, and the centre sphere velocity $U_{0}$. Of course, as $s \rightarrow \infty, U_{0} \rightarrow 1$ and $U_{1} \rightarrow 0$; and as $s \rightarrow 2, U_{0} \rightarrow U_{1}$ as the three spheres move as a solid object. These results for $2 \leq s \leq 3$ are shown in figure 3 . In this case, SD and the current method agree to within graphical accuracy at all separations.

\subsection{Stokesian Dynamics: Conclusions}

In every geometry, we find that SD is highly accurate for well-separated particles. The interesting study occurs when the particles are relatively close - say, within half a radius gap between their surfaces. 


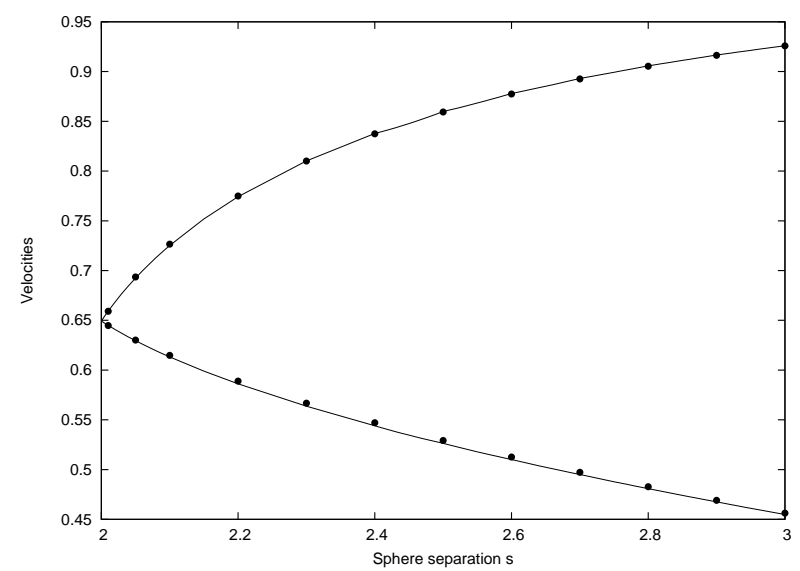

Figure 3: Velocities for three equispaced spheres in a line, with the centre sphere forced towards one of the others, plotted against dimensionless separation. The upper line is the velocity of the centre sphere (which tends to 1 for well-separated spheres) and the lower line that of the outer two spheres. The points $\bullet$ are from the current method, and the curves from Stokesian Dynamics.

Our third test case, in which the spheres were moving in direct approach to (or separation from) one another, is one where the two-sphere lubrication interactions are very strong, so it is hardly surprising that Stokesian Dynamics does very well here. The triangular geometries throw up more surprises.

The out-of-plane triangular motion (almost a rigid motion, apart from the sphere rotations) is captured reasonably well; the inability of SD to fully capture the three-sphere interaction when the spheres are close causes only minor errors. There are no strong lubrication interactions here, so the twobody approximation for close spheres is not as good as in the straight line problem above; but it does well nonetheless.

However, in the in-plane triangular motion we have a test case which shows some genuinely erroneous behaviour in SD. At a worst case, relative errors of over $25 \%$ are observed in the angular velocities. The absolute errors are never very large, but it is clear that this geometry somehow highlights any weakness inherent in SD. It is not surprising, given the relative motion and the strong lubrication interactions, that very close spheres are well modelled in this case. The errors occur in the intermediate regime where the spheres are neither very close nor well separated. It is perhaps surprising that a 


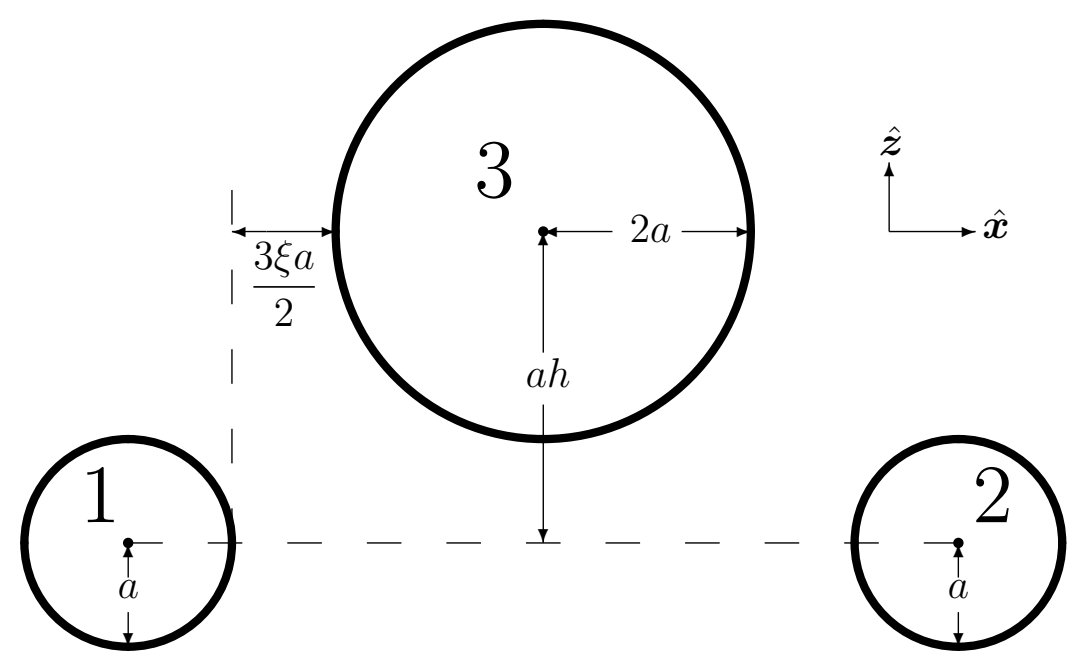

Figure 4: Geometry for section 5. A force $12 \pi \mu a \hat{\boldsymbol{z}}$ is applied to sphere 3.

separation of 0.1 radius should be in the intermediate rather than the close regime; this is where we found the most significant errors.

\section{System with Different Sized Spheres}

Here we consider a family of problems involving two spheres of equal radius $a$ and a third sphere of radius $2 a$. The two spheres of radius $a$ are located with their centres a distance $(6+3 \xi) a$ apart, and the third sphere, of radius $2 a$, lies in the plane of symmetry between them (so that the three sphere centres define an isosceles triangle), a perpendicular distance $a h$ from the line joining the two smaller spheres. A force of magnitude $6 \pi \mu(2 a)$ is applied to the large sphere, acting directly away from the base of the triangle. The geometry is illustrated in figure 4 , along with a standardised numbering of the spheres and a left-handed Cartesian basis system.

The resultant sphere velocities are

$$
\begin{array}{lll}
\boldsymbol{U}_{1}=A \hat{\boldsymbol{x}}+B \hat{\boldsymbol{z}} & \boldsymbol{U}_{2}=-A \hat{\boldsymbol{x}}+B \hat{\boldsymbol{z}} & \boldsymbol{U}_{3}=D \hat{\boldsymbol{z}} \\
\boldsymbol{\Omega}_{1}=-C \hat{\boldsymbol{y}} / a & \boldsymbol{\Omega}_{2}=C \hat{\boldsymbol{y}} / a & \boldsymbol{\Omega}_{3}=\mathbf{0}
\end{array}
$$

with all other components being identically zero by symmetry. We will consider two special cases: the case $\xi=0$, in which the two small spheres have a space exactly $4 a$ between their surfaces, into which the large sphere fits as $h \rightarrow 0$; and the case $h=0$, in which the three spheres lie in a straight line, 
and $A=0$ by symmetry. The contact situation $h=\xi=0$ is a limiting case of both these geometries.

\subsection{Far-field}

When the spheres are well-separated ( $h$ or $\xi$ large) we can predict the leading-order contributions to the velocities. The large sphere, moving under a force of magnitude $6 \pi \mu(2 a)$, where $2 a$ is its radius, will have unit velocity in the $\hat{\boldsymbol{z}}$ direction. The leading approximation to the small sphere velocities is found from the flow field produced by a point force of that magnitude, assuming that the small spheres are simply advected (and rotated) passively with the fluid around them. The flow field and angular velocity due to a point force of magnitude $12 \pi \mu a$ in the $\hat{\boldsymbol{z}}$ direction are

$$
\boldsymbol{u}=\frac{3 a}{r} \cos \theta \boldsymbol{e}_{r}-\frac{3 a}{2 r} \sin \theta \boldsymbol{e}_{\theta}, \quad \boldsymbol{\omega}=\frac{1}{2} \boldsymbol{\nabla} \times \boldsymbol{u}=\frac{3 a}{2 r^{2}} \sin \theta \boldsymbol{e}_{\phi},
$$

where $(r, \theta, \phi)$ are spherical polar coordinates centred on the large sphere, with the axis $\theta=0$ aligned with $\hat{\boldsymbol{z}}$.

Substituting in the position of each small sphere, we obtain (with the upper sign denoting sphere 1 and the lower, sphere 2):

$$
\boldsymbol{U}= \pm \frac{9 a^{3} h(2+\xi)}{4 r^{3}} \hat{\boldsymbol{x}}+\frac{3 a^{3}\left[8 h^{2}+9(2+\xi)^{2}\right]}{8 r^{3}} \hat{\boldsymbol{z}} \quad \boldsymbol{\Omega}=\mp \frac{9 a^{2}(2+\xi)}{4 r^{3}} \hat{\boldsymbol{y}}
$$

with $r^{2}=a^{2}\left(h^{2}+(3+(3 \xi / 2))^{2}\right)$, which gives the following leading-order contributions to the velocity components:

$$
\begin{array}{lllll}
\xi=0, h \rightarrow \infty: & A \sim \frac{9}{2} h^{-2} & B \sim 3 h^{-1} & C \sim \frac{9}{2} h^{-3} & D \rightarrow 1, \\
h=0, \xi \rightarrow \infty: & A \equiv 0 & B \sim \xi^{-1} & C \sim \frac{2}{3} \xi^{-2} & D \rightarrow 1,
\end{array}
$$

all of which we reproduce correctly.

\subsection{Contact}

The contact case $h=\xi=0$ is the limit of both our geometries. We know that at contact the spheres must move as a single rigid object; by symmetry, there will be no angular velocity, so we have only one variable, the linear velocity of the triplet which we denote

$$
\boldsymbol{U}_{1}=\boldsymbol{U}_{2}=\boldsymbol{U}_{3}=V_{C} \hat{\boldsymbol{z}}
$$


This means that at contact we will have $B=D=V_{C}$ and $C=0$.

As we approach contact, we feel the limitations of the Method of Reflections, and we cannot simulate an exactly touching system. Because of memory constraints, we cannot keep more than 150 terms in each angular expansion. This limits the accuracy of our solutions for gaps closer than around $0.01 a$; however, we have iterated until convergence (a neglected contribution per iteration below $10^{-6}$ ) in all cases.

In order to obtain an estimate of $V_{C}$, we observe that in the pure contact limit it does not matter whether the force is applied to the central sphere (sphere 3), or symmetrically to the two end spheres, or indeed in any combination which has the same total force and no applied torque across the rigid body. Thus we consider two scenarios, which must be equivalent at contact:

1. Force $12 \pi \mu a \hat{\boldsymbol{z}}$ applied to the large sphere: resultant velocities $U_{1}^{L} \hat{\boldsymbol{z}}$, $U_{3}^{L} \hat{\boldsymbol{z}}, U_{1}^{L} \hat{\boldsymbol{z}}$

2. Force $6 \pi \mu a \hat{\boldsymbol{z}}$ applied to each small sphere: resultant velocities $U_{1}^{S} \hat{\boldsymbol{z}}$, $U_{3}^{S} \hat{\boldsymbol{z}}, U_{1}^{S} \hat{\boldsymbol{z}}$.

In fact $U_{3}^{S}=U_{1}^{L}$. Then, using the linearity of Stokes flow, it is straightforward to show that a combination of input forces (with the same total force as our two test cases)

$$
\boldsymbol{F}_{1}=\boldsymbol{F}_{2}=\alpha 6 \pi \mu a \hat{\boldsymbol{z}} \quad \boldsymbol{F}_{3}=(1-\alpha) 12 \pi \mu a \hat{\boldsymbol{z}}
$$

would produce velocities

$$
\boldsymbol{U}_{1}=\boldsymbol{U}_{2}=\left(\alpha U_{1}^{S}+(1-\alpha) U_{1}^{L}\right) \hat{\boldsymbol{z}} \quad \boldsymbol{U}_{3}=\left(\alpha U_{3}^{S}+(1-\alpha) U_{3}^{L}\right) \hat{\boldsymbol{z}}
$$

so that if we were to select $\alpha=\left(U_{3}^{L}-U_{1}^{L}\right) /\left(U_{1}^{S}+U_{3}^{L}-2 U_{1}^{L}\right)$ we would have

$$
\boldsymbol{U}_{1}=\boldsymbol{U}_{2}=\boldsymbol{U}_{3}=\left(\frac{U_{1}^{S} U_{3}^{L}-\left(U_{1}^{L}\right)^{2}}{U_{1}^{S}+U_{3}^{L}-2 U_{1}^{L}}\right) \hat{\boldsymbol{z}}
$$

We have calculated these velocities, and the resultant estimate for $V_{C}$, at various values of $\xi$ in the case $h=0$, and these results are shown in table 4 . All gaps $\xi \leq 0.01$ predict $V_{C}=0.800$ correct to 3 decimal places, so this is our result for contact.

\subsection{Moderate separations}

In figures 5 and 6 we show the velocities $A, B, C$ and $D$ as either $h$ or $\xi$ is varied. The plots against $h$ are on a linear scale, whereas those against $\xi$ 


$\begin{array}{ccccc}\xi & U_{1}^{S} & U_{3}^{S}=U_{1}^{L} & U_{3}^{L} & V_{C} \text { estimate } \\ 0.5000 & 1.0800821 & 0.44874295 & 0.99573622 & 0.741817 \\ 0.2000 & 1.0636104 & 0.53144811 & 0.98659225 & 0.776773 \\ 0.1000 & 1.0432806 & 0.57179602 & 0.97712348 & 0.789751 \\ 0.0500 & 1.0221154 & 0.59954901 & 0.96690727 & 0.796066 \\ 0.0200 & 0.9968676 & 0.62468302 & 0.95370717 & 0.799321 \\ 0.0100 & 0.9805922 & 0.63853121 & 0.94447949 & 0.800030 \\ 0.0050 & 0.9666076 & 0.64965058 & 0.93606716 & 0.800108 \\ 0.0020 & 0.9512996 & 0.66127634 & 0.92661956 & 0.799839\end{array}$

Table 4: Linear velocities of the outer spheres $\left(U_{1}\right)$ and the central, larger sphere $\left(U_{3}\right)$ for two different distributions of the force $12 \pi \mu a \hat{\boldsymbol{z}}$ along the triplet of spheres at $h=0$ : force applied to the large sphere $\left(U^{L}\right)$ or distributed equally between the two small spheres $\left(U^{S}\right)$. The final column is the resultant estimate of the common contact velocity $V_{C}$.

are on a log scale: this is simply to present two different perspectives, as the two sets of curves look rather similar to one another.

At a first glance, figure 5 seems to indicate that we have a problem at small separations, as the points for $A$ and $C$ are not obviously tending to zero as $h \rightarrow 0$, and those for $B$ and $D$ are not obviously tending to a common value of 0.800 . However, this is purely a result of the slow logarithmic dependence of these velocities on inter-particle separation. This issue is made explicit in the logscale figure, figure 6 .

In this logscale plot against $\xi$ (figure 6), we also give far- and near-field approximations to the velocities. For the far field, these are the forms derived in equation (36). We could, of course, have plotted the equivalent forms of equation (37) in figure 5, but they are not good fits on the scale of that figure. For the near field, we use a semi-empirical curve for each velocity for small gaps, whose derivation we explain here. These curves use the extracted contact velocities $B=D=V_{C}=0.800$ and the fact that $C=0$ at contact, and are based on the observation that the particle interactions are likely to be dominated by the pair-interactions between particles 1 and 3 and particles 2 and 3 ; that is, direct interactions between the two small particles will be less important. If we were to discard sphere 2 , the resultant velocities for 


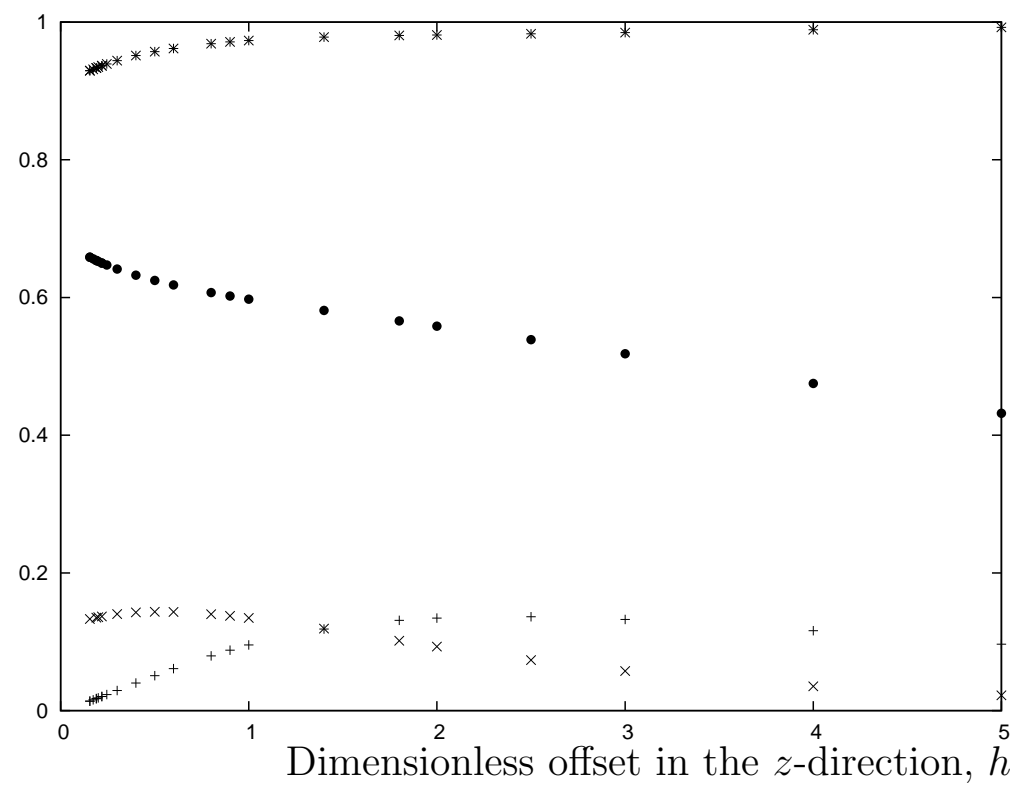

Figure 5: Plot of the velocities $A(+), B(\bullet), C(\times)$ and $D(*)$ against $h$ in the case $\xi=0$. In the contact limit $h=0$, we expect $A=C=0$ and $B=D=0.800$.

small $\xi$ are given $[15,16]$ by

$$
\begin{aligned}
& \boldsymbol{U}_{1}=\frac{4}{3} \frac{0.53482\left(\ln \xi^{-1}\right)^{2}+2.50225 \ln \xi^{-1}+1.23963}{\left(\ln \xi^{-1}\right)^{2}+5.59906 \ln \xi^{-1}+4.17702} \hat{\boldsymbol{z}}+O\left(\xi(\ln \xi)^{3}\right)(38) \\
& \boldsymbol{U}_{3}=\frac{0.92729\left(\ln \xi^{-1}\right)^{2}+5.61052 \ln \xi^{-1}+4.40223}{\left(\ln \xi^{-1}\right)^{2}+5.59906 \ln \xi^{-1}+4.17702} \hat{\boldsymbol{z}}+O\left(\xi(\ln \xi)^{3}\right) \\
& \boldsymbol{\Omega}_{1}=\frac{4}{3 a} \frac{-0.05355\left(\ln \xi^{-1}\right)^{2}-0.99178 \ln \xi^{-1}+0.04487}{\left(\ln \xi^{-1}\right)^{2}+5.59906 \ln \xi^{-1}+4.17702} \hat{\boldsymbol{y}}+O(\xi \ln \xi)(40) \\
& \boldsymbol{\Omega}_{3}=-\frac{3}{4 a} \frac{0.09519\left(\ln \xi^{-1}\right)^{2}-0.03922 \ln \xi^{-1}-0.23881}{\left(\ln \xi^{-1}\right)^{2}+5.59906 \ln \xi^{-1}+4.17702} \hat{\boldsymbol{y}}+O(\xi \ln \xi)(.41)
\end{aligned}
$$

We expect both the logarithmic dependence on gap, and the specific denominator of the fractions, to carry over to our three-sphere problem. So we fit the form

$$
U=\frac{\alpha\left(\ln \xi^{-1}\right)^{2}+\beta \ln \xi^{-1}+\gamma}{\left(\ln \xi^{-1}\right)^{2}+5.59906 \ln \xi^{-1}+4.17702}
$$

to each of our scalar velocities $B, C$ and $D$, forcing the known contact values 


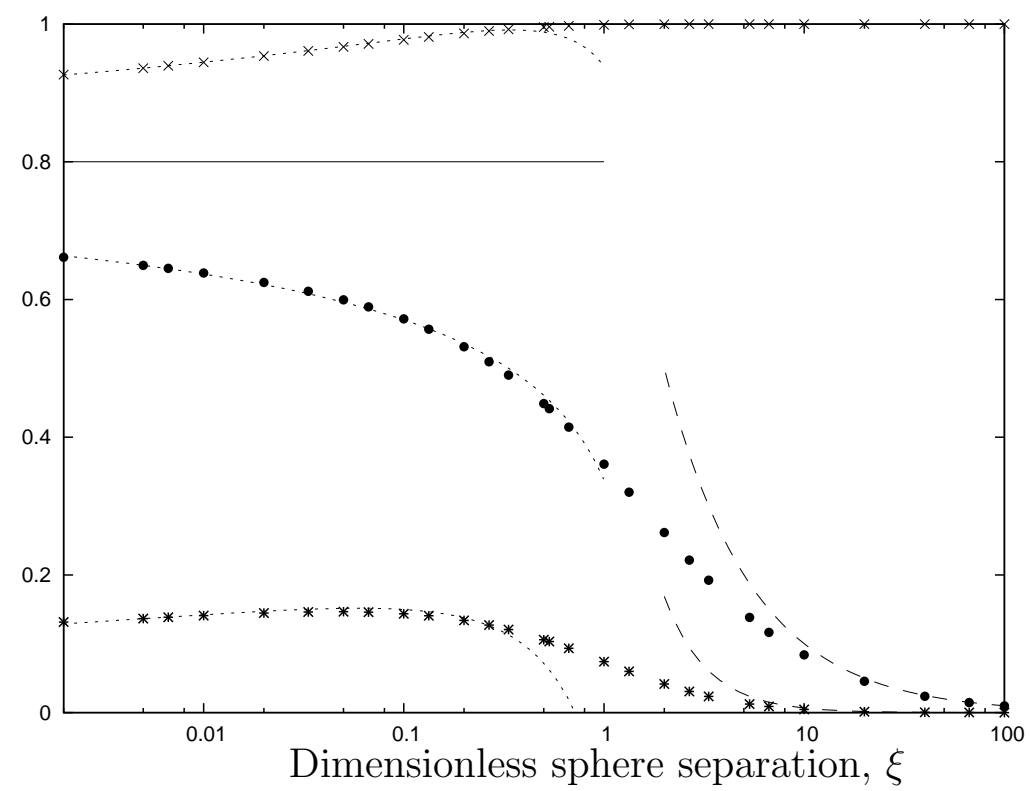

Figure 6: Plot of the non-zero velocities $B(\bullet), C(\times)$ and $D(*)$ against $\xi$ in the case $h=0$. The two dashed lines on the right of the plot (plotted only for $\xi \geq 2$ ) are the far-field forms for $B$ and $C$ from equation (36); in the far field $D \rightarrow 1$. The dotted lines on the left of the plot (plotted only for $\xi \leq 1$ ) are the semi-empirical fit of equations (43-45). The horizontal line is the extrapolated contact velocity $V_{C}$, which is also the contact limit of $B_{n f}$ and $D_{n f}$.

$\alpha=V_{C}, 0, V_{C}$ respectively. The near field fits we obtain are

$$
\begin{aligned}
B_{n f} & \sim \frac{0.800\left(\ln \xi^{-1}\right)^{2}+3.086 \ln \xi^{-1}+1.413}{\left(\ln \xi^{-1}\right)^{2}+5.59906 \ln \xi^{-1}+4.17702} \\
C_{n f} & \sim \frac{1.701 \ln \xi^{-1}-0.566}{\left(\ln \xi^{-1}\right)^{2}+5.59906 \ln \xi^{-1}+4.17702} \\
D_{n f} & \sim \frac{0.800\left(\ln \xi^{-1}\right)^{2}+5.960 \ln \xi^{-1}+3.924}{\left(\ln \xi^{-1}\right)^{2}+5.59906 \ln \xi^{-1}+4.17702}
\end{aligned}
$$

and these are included on the left-hand-side of figure 6 . It is clear that the data fit well with this model, so the appearance of non-convergence to the known contact values is simply an artifact of the log-form of the mobilities.

We see that, as expected, in either geometry the velocity $B$ of the small spheres increases monotonically from zero in the far field, to 0.800 at contact, 
as the spheres are brought closer together and the small spheres feel more of the force being applied to the large sphere.

The velocity $D$ of the large sphere, similarly, decreases monotonically as it is slowed by the presence of the smaller spheres, from 1 at very large separations, to 0.800 at contact; but even for relatively close spheres its velocity is still well approximated by the velocity for an isolated sphere.

The remaining velocities $A$ and $C$, which are both zero at contact and also when the particles are very well separated, each therefore have a maximum at some intermediate separation. For the antisymmetric velocities $A$, the maximum velocity magnitude in the triangular geometry is 0.136 (at a position $h=2.5$ ); in the straight line geometry these velocities are identically zero. For the angular velocities $C$, the maximum velocity magnitude in the triangular geometry is 0.143 (at a position $h=0.5$, which represents a surface-to-surface gap of $0.041 a)$; and in the straight line geometry, the maximum velocity is 0.146 (at a position $\xi=0.05$, which represents a surface-to-surface gap of $0.075 a$ ). The close location of these maxima in the angular velocities is an indication of how challenging the near-field solution of this problem can be.

In isolation, this example gives only an idea of the interactions required to properly model a flow containing polydisperse spheres; but the computer program used to produce these data is available from the author by email on request.

\section{Discussion}

We have presented a numerical method for the calculation of the motion of three spheres in Stokes flow which is a slight modification to the standard Method of Reflections, incorporating rotation of coefficient fields by an efficient method introduced by [20]. The method has the potential to be more accurate than multipole methods, but because of its numerical cost is unsuitable for problems involving more than three spheres. We observe that the iterative scheme converges unconditionally for the mobility problem, whereas the corresponding resistance problems often fail to converge when spheres are close.

After validating our method against the existing literature, we first compared Stokesian Dynamics with our results for a few simple test cases. In most cases, as would be expected, Stokesian Dynamics is very accurate, but 
it was possible to find scenarios producing errors of up to $25 \%$. These most serious errors occurred at an inter-particle separation of around 0.1 radius.

Finally, we have produced a case study of a system having spheres of different sizes: specifically, two spheres of radius $a$ and one of radius $2 a$. In the specific geometry we studied, we found that the angular velocities of the small spheres (which would be zero at contact) had a maximum value when the surface gap between the particles was around $0.05 a$, indicating that lubrication theory in this instance is liable to produce a good approximation to the true velocity only for spheres which are exceptionally close to contact. For the simple case of the three spheres, arranged symmetrically, contacting in a straight line, and feeling a force perpendicular to their line of centres, we calculated the drag coefficient for the triplet as

$$
\frac{U}{F}=\frac{0.800}{12 \pi \mu a}=\frac{0.0667}{\pi \mu a} .
$$

The FORTRAN code used to produce all the results in this paper is available from the author on request by email.

\section{Acknowledgments}

The author would like to thank Alexander Zinchenko and Sangtae Kim for helpful discussions.

The initial stages of this work were carried out at the University of Colorado Boulder when the author was a research associate in the group of Prof. R. H. Davis and supported by the National Science Foundation grant CTS-9416702.

\section{References}

[1] J. Happel, H. Brenner, Low Reynolds number hydrodynamics, Marinus Nijhoff Publishers, The Hague, 1967.

[2] I. L. Claeys, J. F. Brady, Suspensions of prolate spheroids in Stokes flow. part 1. dynamics of a finite number of particles in an unbounded fluid, J. Fluid Mech. 251 (1993) 411-442.

[3] I. L. Claeys, J. F. Brady, Suspensions of prolate spheroids in Stokes flow. part 2. statistically homogeneous dispersions, J. Fluid Mech. 251 (1993) 443-477. 
[4] D. Liu, E. E. Keaveny, M. R. Maxey, G. E. Karniadakis, Force-coupling method for flows with ellipsoidal particles, Journal of Computational Physics 228 (2009) 3559-3581.

[5] M. A. v. d. Hoef, R. Beetstra, J. A. M. Kuipers, Lattice-Boltzmann simulations of low-Reynolds-number flow past mono- and bidisperse arrays of spheres: results for the permeability and drag force, J. Fluid Mech. 528 (2005) 233-254.

[6] M. Abbas, E. Climent, O. Simonin, M. R. Maxey, Dynamics of bidisperse suspensions under Stokes flows: Linear shear flow and sedimentation, Physics of Fluids 18 (2006) 121504.

[7] G. J. Wagner, N. Moës, W. K. Liu, T. Belytschko, The extended finite element method for rigid particles in Stokes flow, International Journal for Numerical Methods in Engineering 51 (2001) 293-313.

[8] T. Tran-Cong, N. Phan-Thien, Stokes problems of multiparticle systems: A numerical method for arbitrary flows, Physics of Fluids A: Fluid Dynamics 1 (1989) 453-461.

[9] M. S. Ingber, Dynamic simulation of the hydrodynamic interaction among immersed particles in Stokes flow, International Journal for $\mathrm{Nu}-$ merical Methods in Fluids 10 (1990) 791809.

[10] B. Cichocki, R. B. Jones, R. Kutteh, E. Wajnryb, Friction and mobility for colloidal spheres in Stokes flow near a boundary: The multipole method and applications, J. Chem. Phys. 112 (2000) 2548-2561.

[11] J. F. Brady, G. Bossis, Stokesian Dynamics, Ann. Rev. Fluid Mech. 20 (1988) 111-157.

[12] M. Abramowitz, I. Stegun (Eds.), Handbook of Mathematical Functions, Dover, 9th edition, 1972.

[13] A. Z. Zinchenko, Effective conductivity of loaded granular materials by numerical simulation, Phil. Trans. R. Soc. Lond. A 356 (1998) 29532998.

[14] E. W. Hobson, Spherical and Ellipsoidal Harmonics, Cambridge University Press, 1931. 
[15] D. J. Jeffrey, Y. Onishi, Calculation of the resistance and mobility functions for two unequal rigid spheres in low-Reynolds-number flow, J. Fluid Mech. 139 (1984) 261-290.

[16] S. Kim, S. J. Karrila, Microhydrodynamics: Principles and selected applications, Butterworth-Heinemann, 1991.

[17] S. Kim, Stokes flow past three spheres: an analytic solution, Phys. Fluids 30 (1987) 2309-2314.

[18] I. A. Lasso, P. D. Weidman, Stokes drag on hollow cylinders and conglomerates, Phys. Fluids 29 (1986) 3921-3934.

[19] P. Ganatos, R. Pfeffer, S. Weinbaum, A numerical-solution technique for three-dimensional Stokes flows, with application to the motion of strongly interacting spheres in a plane, Journal of Fluid Mechanics 84 (1978) $79-111$.

[20] A. Z. Zinchenko, An efficient algorithm for calculating multiparticle thermal interaction in a concentrated dispersion of spheres, J. Comp. Phys. 111 (1994) 120-135. 